Methods: We report two cases of successful intra-lesional injections of sodium thiosulfate (STS).

Results: Case 1: A 44-years old woman, with history of dermatomyositis developed in 2009 several TC involving the extensor surfaces of the right elbow and forearm, the two ischiatic regions, fingers and lumbar back. As TC kept growing despite successive treatments, including intravenous STS, we proposed weekly intra-lesional injections of $10 \%$ STS in the elbow's lesion. The injected volume varied from 10 to $30 \mathrm{~mL}$ at each session. Fourrier transform infrared spectroscopy (FTIR) of the aspirations confirmed that TC was composed of carbonated-apatite crystals. After 6-month treatment, we observed clinical and radiological regression of elbow TC whereas bottom TC and finger calcifications were unchanged (Fig 1). No side effect was observed except a subcutaneous infection occurring after an injection during the fifth month. This infection resolved with anti-staphycoccal antibiotics. Calcium, bicarbonate and chlorus serum levels, anion gap and eGFR remained unchanged during STS treatment.

Case 2: A 42-year old male presented with a prolonged history of hyperphosphatemic familial TC, confirmed by genetic analysis revealing homozygous mutations in the gene encoding the fibroblast growth factor 23. The extraosseous calcifications comprised a plain lesion on the right side of the left tibia and a massive heterogeneous lesion in the right buttock, making it the sitting position impossible. Treatment with maximal dose of phosphate binders, diet prescription and an attempt to surgically remove of the buttock's lesion were unsuccessful. Topical application of STS led to a near complete disappearance of the tibial lesion but was inefficient to treat the TC at the buttock. Considering the potential efficacy of STS in this patient, local injections of $25 \%$ STS (12 mL every week) were performed. The calcified material aspiration's analysis confirmed that TC was composed of carbonated-apatite crystals. Calcium and bicarbonate serum levels, anion gap and eGFR remained unchanged. After a 12-month treatment of STS injections, the lesion had significantly regressed (Fig 2), allowing the patient to sit again with no pain.
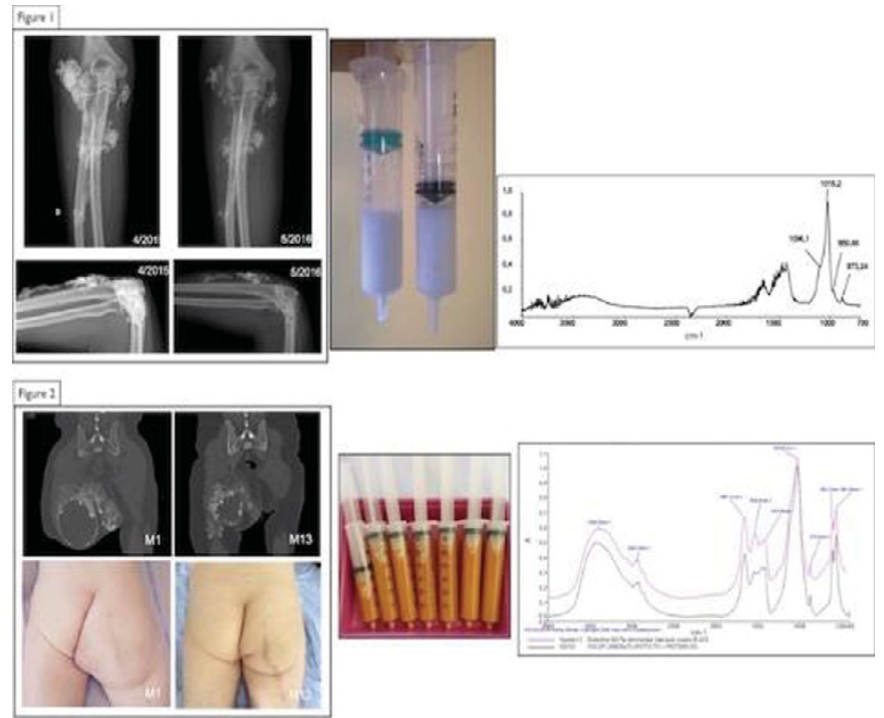

Conclusions: Intra-lesional injection of STS seems to be a promising treatment for TC. More studies are needed to confirm these results, and to understand the mechanisms implicated in the calcinosis resorption.

Disclosure of Interest: None declared

DOI: 10.1136/annrheumdis-2017-eular.2809

\section{AB0883 THE SENSITIVITY OF GOUT SPECIFIC ULTRASOUND SIGNS AT METATARSOPHALANGEAL JOINTS WOULD BE BETTER BY THE DORSAL SURFACE EXAMINATION}

M.A. Mahdi ${ }^{1}$, H. Rkain ${ }^{1}$, M. Erraoui ${ }^{1}$, R. Watfeh ${ }^{2}$, S. Aktaou ${ }^{1}$, L. Tahiri ${ }^{1}$, R. Bahiri ${ }^{1}$, F. Allali ${ }^{1}$, N. Hajjaj-Hassouni ${ }^{1}$. ${ }^{1}$ Rheumatology; ${ }^{2}$ Faculty of Medicine and Pharmacy, University Mohamed V, Rabat, Morocco

Objectives: To compare the prevalence of ultrasonographic gout specific signs at the dorsal and plantar surfaces of the metatarsophalangeal joints (MTP).

Methods: This is a cross-sectional study which includes 15 patients with chronic gout, defined according to the American College of Rheumatology criteria (ACR 1977). Ultrasound (US) examination was performed using a high-frequency linear probe (Toshiba Xario ${ }^{\circledR}$, frequency $(8-14 \mathrm{MHz})$ ) in B mode. 150 articular sites were studied at their dorsal and plantar surfaces. The ultrasound has objectified the presence of two signs: hyperechoic band over the superficial margin of the articular cartilage described as a double contour (DC) and the tophaceous deposits at the joint cavity. We compared the prevalence of the two signs between the dorsal and palmar surfaces at each site studied.

Results: The mean age at onset was $54.7 \pm 12,6$ years, and the median diagnosis duration was $0(0.3)$ years)
The results of the US examination are summarized in Table 1.

Table 1. Prevalence comparison of DC and tophaceous deposits between dorsal and plantar surfaces at MTP joints

\begin{tabular}{lccccccc}
\hline Joints $(\mathrm{N}=150)$ & \multicolumn{3}{c}{ Double contour $(\%)$} & & \multicolumn{3}{c}{ Tophaceous deposits $(\%)$} \\
\cline { 2 - 3 } \cline { 7 - 8 } & Dorsal surface & Plantar surface & $\mathrm{P}$ & & Dorsal surface Plantar surface & $\mathrm{P}$ \\
\hline MTP 1 $(\mathrm{N}=30)$ & 33,3 & 10 & 0,03 & 56,7 & 6,7 & 0,01 \\
MTP 2 $(\mathrm{N}=30)$ & 13,3 & 0 & $<0.001$ & 13,3 & 0 & $<0.001$ \\
MTP 3 $(\mathrm{N}=30)$ & 6,7 & 0 & $<0.001$ & 3,3 & 0 & $<0.001$ \\
MTP 4 $(\mathrm{N}=30)$ & 0 & 0 & $<0.001$ & 6,7 & 0 & $<0.001$ \\
MTP 5 $(\mathrm{N}=30)$ & 3,3 & 0 & $<0.001$ & 10 & 0 & $<0.001$ \\
\hline
\end{tabular}

Conclusions: Our study suggests that globally, DC predilect significatily in dorsal than in plantar surfaces of MTP joints. These results should be verified on a larger population.

Disclosure of Interest: None declared

DOI: 10.1136/annrheumdis-2017-eular.3773

\section{AB0884 PREVALENCE OF ULTRASONOGRAPHIC GOUT SPECIFIC SIGNS OF HAND AND FINGERS JOINTS}

M.A. Mahdi ${ }^{1}$, H. Rkain ${ }^{1}$, M. Erraoui ${ }^{1}$, R. Watfeh ${ }^{2}$, S. Aktaou ${ }^{1}$, L. Tahiri ${ }^{1}$, R. Bahiri ${ }^{1}$, F. Allali ${ }^{1}$, N. Hajjaj-Hassouni ${ }^{1} .{ }^{1}$ Rheumatology; ${ }^{2}$ Faculty of Medicine and Pharmacy, University Mohamed V, Rabat, Morocco

Objectives: To evaluate the prevalence of ultrasonographic gout specific signs of hand and fingers joints.

Methods: This is a cross-sectional study which includes 15 patients with chronic gout, defined according to the American College of Rheumatology criteria (ACR 1977). Ultrasound (US) examination was performed using a high-frequency linear probe (Toshiba Xario ${ }^{\circledR}$, frequency $(8-14 \mathrm{MHz})$ ) in B mode. 540 articular sites were studied at their dorsal surface. The ultrasound has objectified the presence of two signs: hyperechoic band over the superficial margin of the articular cartilage described as a double contour (DC) and the tophaceous deposits at the joint cavity.

Results: The mean age at onset was $54.7 \pm 12,6$ years, and the median diagnosis duration was 0 (0.3) years)

The results of the US examination are summarized in Table 1.

Table 1. Prevalence of ultrasonographic gout specific signs of the wrist, MCP, PIP and DIP joints in the studied population

\begin{tabular}{lcc}
\hline Joints $(\mathrm{N}=540)$ & Double contour $(\%)$ & Tophaceous deposits $(\%)$ \\
\hline Wrist joints $(N=120)$ & 12,6 & 36 \\
Radiocarpal $(\mathrm{N}=30)$ & 20 & 43,3 \\
Ulnocarpal $(\mathrm{N}=30)$ & 13,3 & 50 \\
Scaphotrapezial $(\mathrm{N}=30)$ & 3,3 & 16,7 \\
Trapeziometacarpal $(\mathrm{N}=30$ & 13,3 & 43,3 \\
MCP $(\mathrm{N}=150)$ & 8 & 18 \\
MCP 1 $(\mathrm{N}=30)$ & 3,3 & 16,7 \\
MCP 2 $(\mathrm{N}=30)$ & 13,3 & 33,3 \\
MCP 3 $(\mathrm{N}=30)$ & 6,7 & 16,7 \\
MCP 4 $(\mathrm{N}=30)$ & 6,7 & 3,3 \\
MCP 5 $(\mathrm{N}=30)$ & 10 & 20 \\
PIP $(N=150)$ & 4 & 16,6 \\
IP $(\mathrm{N}=30)$ & 3,3 & 30 \\
PIP 2 $(\mathrm{N}=30)$ & 6,7 & 16,7 \\
PIP 3 $(\mathrm{N}=30)$ & 6,7 & 20 \\
PIP 4 $(\mathrm{N}=30)$ & 3,3 & 10 \\
PIP 5 $(\mathrm{N}=30)$ & 0 & 6,7 \\
DIP $(N=120)$ & 0,8 & 10 \\
DIP 2 $(\mathrm{N}=30)$ & 0 & 13,3 \\
DIP 3 $(\mathrm{N}=30)$ & 3,3 & 13,3 \\
DIP 4 $(\mathrm{N}=30)$ & 0 & 6,7 \\
DIP 5 $(\mathrm{N}=30)$ & 0 & 6,7
\end{tabular}

Conclusions: This study showed a predilection for the gout specific ultrasound signs (DC and tophaceous deposits) of the wrist and MCP joints. The contribution of musculoskeletal ultrasound seems to be very interesting to objectify the presence of gout specific signs in the hand and fingers joints.

Disclosure of Interest: None declared

DOI: 10.1136/annrheumdis-2017-eular.3877

\section{AB0885 PREVALENCE OF ULTRASONOGRAPHIC GOUT SPECIFIC SIGNS OF FOOT JOINTS}

M.A. Mahdi ${ }^{1}$, H. Rkain ${ }^{1}$, M. Erraoui ${ }^{1}$, R. Watfeh ${ }^{2}$, S. Aktaou ${ }^{1}$, L. Tahiri ${ }^{1}$, R. Bahiri ${ }^{1}$, F. Allali ${ }^{1}$, N. Hajjaj-Hassouni ${ }^{1}$. ${ }^{1}$ Rheumatology; ${ }^{2}$ Faculty of Medicine and Pharmacy, University Mohamed V, Rabat, Morocco

Objectives: To evaluate the prevalence of ultrasonographic gout specific signs of foot joints.

Methods: This is a cross-sectional study which includes 15 patients with chronic gout, defined according to the American College of Rheumatology criteria (ACR 1977). Ultrasound (US) examination was performed using a high-frequency linear probe (Toshiba Xario ${ }^{\circledR}$, frequency $(8-14 \mathrm{MHz})$ ) in B mode. 330 articular sites were 